\title{
How can mitochondrial DNA deletions act as a biomarker for the detection of endometriosis within the clinic?
}

\author{
Jennifer M Creed*,1, Andrea Maggrah¹, Robert Usher ${ }^{1}$, Elise Desa ${ }^{1}$ \& Andrew Harbottle ${ }^{1}$ \\ ${ }^{1}$ MDNA Life Sciences Inc., 2054 Vista Parkway, Ste 400, West Palm Beach, FL 33411, USA \\ *Author for correspondence: j.creed@mdnalifesciences.com
}

\begin{abstract}
' these features of the mitochondrial genome, its mutated subtypes and especially large-scale deletions, potentially resolve limitations of endometriosis biomarkers of different origins and offer the promise of successful translation to clinical application."
\end{abstract}

First draft submitted: 4 October 2019; Accepted for publication: 18 October 2019; Published online: 5 November 2019

Keywords: biomarker $\bullet$ DNA deletion $\bullet$ endometriosis $\bullet$ mitochondrial DNA • molecular diagnostic $\bullet$ mtDNA

Endometriosis is common, affecting 5-10\% of females of child-bearing age, with many enduring significant quality of life issues. Several metrics reflect the diagnostic challenges including the average 9-year delay between symptom presentation and diagnosis, as well as the risk of unnecessary surgery for women with symptoms who ultimately do not have endometriosis (up to 29\%) [1]. Yet, despite increasing awareness within the medical community and significant research investment, few advances have been made to substantially improve the diagnostic pathway.

Not surprisingly, the identification of noninvasive diagnostic aids for endometriosis is noted as a key research priority [2] and blood-based biomarkers present an exciting opportunity to address this need. Biomarkers that can be routinely measured in conventional clinical specimens such as blood offer an inexpensive and new intervention point in the clinical pathway to better steer patients toward optimal diagnostic and treatment decisions. Additionally, where diagnosis is impeded by a reliance on qualitative indicators, biomarkers can provide a welcome quantitative assessment for consideration in the patient's workup.

Unfortunately, despite a now large body of research, noninvasive biomarkers of endometriosis have eluded translation from bench to bedside. The reason for this failure is varied and includes impracticalities of testing, such as for biomarkers affected by the menstrual cycle [3]; poor sensitivity for low stage disease [4]; complex assays causing higher cost and/or failure rate; challenging sample collection requirements such as from menstrual fluid; and lack of reproducibility across multiple studies, as with miRNA dysregulation [5] and TNF- $\alpha$ [6]. These inadequacies can be a result of variability in the limit of detection of the biomarker(s) under physiological conditions.

Recently, J Creed, A Maggrah, B Reguly, and A Harbottle, MDNA Life Sciences Inc. (FL, USA) reported the potential for clinical use of mitochondrial genome mutations for the detection of endometriosis in the plasma of females presenting with symptoms [7]. While not previously reported in this application, mutations of the mitochondrial genome are frequently disease associated. Some are causative and associated with rare mitochondrial disorders characterized by defective oxidative phosphorylation where the causative mutation is often inherited and exists within a heteroplasmic population alongside unaffected wild-type mitochondrial DNA (mtDNA). Other mtDNA mutations are associated with diseases such as cancer where the causative nature of the mutation is less clear, but too exist in a heteroplasmic state.

Each human cell contains a unique complement of nucleic acids, most of which reside in the nucleus in the form of the nuclear genome. Within the cytoplasm, a second, smaller form exists, in both length and mass, as the mitochondrial genome. This mtDNA is a mere $16,568 \mathrm{bp}$, and in contrast to its nuclear counterpart, exists primarily in a circular and supercoiled form in vivo. Until recently, the function of the mitochondrial genome was thought to be limited to its role in energy production, however continued research reveals that the complexity of this 
small DNA was vastly underestimated and roles in disease initiation, progression and intercellular communication are among its critical functions [8].

Typically, tens to hundreds of mtDNAs exist in each cell - in contrast to the one or two copies of the nuclear genome. Thus, while the mass of DNA in a cell is $>99.9 \%$ nuclear in origin, the difference in copy number is significant. While there is a need for cellular investment in maintaining the integrity of each copy of the nuclear genome to avoid potentially catastrophic results, mtDNA, with multiple copies per cell can fulfill its biological mandate even when a percentage is mutated. Additionally, while the nuclear genome is histone-bound and employs a number of DNA repair mechanisms, mtDNA is not associated with histones - though does form protein-DNA complexes called mitochondrial nucleoids - and has a mutation rate $10-17 \times$ that of the nuclear DNA, with mutations becoming detectable early in disease pathogenesis [9].

Given this high mutation rate, it is not surprising that somatic mtDNA mutations associated with various diseases have been widely reported in the literature and include single nucleotide polymorphisms as well as largescale rearrangements [9]. Frequently, these reports show an increase in mutation load in affected tissue, primarily as single nucleotide variants, which if amenable to translation into the clinic, would require the use of tissue obtained surgically or via biopsy. This is perhaps most applicable then, to prognostic applications, following diagnosis. However, large deletions of the mtDNA have been reported associated with specific diseases or predisease conditions [10-12] and detectable in body fluids such as plasma. Remarkably, Fliss et al. report that mutated mtDNA is circulating at quantities $19-220 \times$ that of nuclear DNA [13].

Indeed, mtDNA is not confined to the mitochondrial matrix but rather circulates in biofluids contained within mitochondria that have escaped the cell, within protein-DNA complexes such as nucleoids, or inside or attached to extracellular vesicles, each of which, except the latter affords protection from enzymatic damage such as DNAses [14].

Some mitochondrial genome deletions such as the 4977-bp deletion can be readily detected even in the absence of a defined disease state and are perhaps associated more closely with aging [15], however many report specific mitochondrial deletions are associated with preclinical conditions, precursor lesions and specific tumor types $[10,11,16]$. Previously unreported and novel deletions are technically challenging to identify and thus much of the literature is focused on those widely reported such as the common 4977-bp deletion. However, it is the less ubiquitous deletions that offer the greatest disease specificity and ultimately diagnostic accuracy. Once the novel sequence created by the recircularization of the deleted DNA is characterized, deletions are readily targeted with sequence-specific oligonucleotides.

MtDNA mutations are frequently increased in diseased cells but also in nearby histologically normal appearing cells, termed a field effect. This field effect is especially important in the context of circulating biomarkers as detection is not limited to the mutation originating from only tumor cells but neighboring normal-appearing cells as well. It can be anticipated that the high-copy number of circulating mutated mtDNA is related to this field effect.

Each of these features of the mitochondrial genome, its mutated subtypes and especially large-scale deletions, potentially resolve limitations of endometriosis biomarkers of different origins and offer the promise of successful translation to clinical application. Recently, MDNA Life Sciences introduced the first in vitro diagnostic test to detect a mtDNA deletion in blood plasma to market in the European Union. This test is intended for men with suspicion of clinically significant prostate cancer when PSA is $<10 \mathrm{ng} / \mathrm{ml}$. The successful translation of such an assay to a regulated device required to satisfy standards of robustness, reproducibility and diagnostic accuracy among others attests to the feasibility for a mitochondrial biomarker test for endometriosis.

Building upon the concept of a highly accurate, simple, blood-based test for endometriosis based on a mitochondrial biomarker, these authors foresee several potential applications to significantly improve the current standard of care for endometriosis. A vital need for such a test is in the general practitioner setting where effective patient assessment can be frustrated by the qualitative and varied nature of clinical presentation, especially where pain is the primary symptom. A test at this critical juncture could significantly reduce the current diagnostic delay, particularly if the decision-making threshold to test is low. The test result would then be obtained early in the diagnostic assessment facilitating the more rapid selection of a diagnostic or low-risk treatment pathway. In this setting, a diagnostic cut-off selected for high sensitivity and in turn low false-negative rate would be of greatest benefit as the next step for those with a positive-test result would carry a low risk, such as initiation of treatment with oral contraceptive pill or perhaps imaging to assess extent of disease. Such a use could serve to avoid unnecessary specialist referral, effectively triaging the need for specialists to women with more severe disease. It can be foreseen 
that a reduction in the need for specialists could also be achieved if the incidence of severe disease and infertility are reduced as a direct result of earlier intervention.

Second, patients with a presumptive diagnosis of endometriosis who are considering laparoscopy for treatment purposes can benefit from a confirmatory biomarker test, wherein a diagnostic cut-off selected for high specificity and in turn low false-positive rate could aid in prevention of unnecessary surgery.

Finally, as severity of symptoms poorly parallels the severity of endometriosis, absence of return of symptoms may fail to alert patient and physician to progressing disease, a disconnect that can have a dire effect on pelvic health and fertility. Ongoing monitoring following diagnosis and during treatment may identify those whose disease is progressing in the absence of worsening symptoms. In this scenario a positive test result may be followed by non-invasive imaging and thus a diagnostic cut-off selected for high sensitivity is favored.

MtDNA deletions, through their high-copy number and mutation rate, field effect, disease specificity and biofluid availability, are perhaps uniquely suited to meet the unmet clinical needs in the endometriosis diagnostic pathway and provide a practical opportunity to lessen the time to diagnosis and treatment, reduce unnecessary surgeries and provide a guardrail against undetected disease progression.

\section{Author contributions}

All authors contributed to drafting of this manuscript.

\section{Financial \& competing interests disclosure}

J Creed, A Maggrah, R Usher, E Desa and A Harbottle are employed by MDNA Life Sciences Inc. and/or are inventors of intellectual property including patent applications relating to the information disclosed herein. The authors have no other relevant affiliations or financial involvement with any organization or entity with a financial interest in or financial conflict with the subject matter or materials discussed in the manuscript apart from those disclosed.

No writing assistance was utilized in the production of this manuscript.

\section{Open access}

This work is licensed under the Attribution-NonCommercial-NoDerivatives 4.0 Unported License. To view a copy of this license, visit http://creativecommons.org/licenses/by-nc-nd/4.0/

\section{References}

1. Schliep KC, Stanford JB, Zhen C et al. Interrater and intrarater reliability in the diagnosis and staging of endometriosis. Obstet. Gynecol. 120(1), 104-112 (2012).

2. Mayor S. Endometriosis research should aim to improve diagnosis and halt progression, meeting hears. BMJ 357, j2438 (2017).

3. Berker B, Seva M. Problems with the diagnosis of endometriosis. Womens Health 11(5), 597-601 (2015).

4. Mol BW, Bayram N, Lijmer JG. The performance of CA-125 measurement in the detection of endometriosis: a meta-analysis. Fertil. Steril. 70(6), 1101-1108 (1998).

5. Agrawal S, Tapmeier T, Rahmioglu N, Kirtley S, Zondervan K, Becker C. The miRNA mirage: how close are we to finding a non-invasive diagnostic biomarker in endometriosis? a systematic review. Int. J. Mol. Sci. 19(2), (2018).

6. Kennedy SH, May KE, Conduit-Hulbert SA, Villar J, Kirtley S, Becker CM. Peripheral biomarkers of endometriosis: a systematic review. Hum. Reprod. Update 16(6), 651-674 (2010).

7. Creed JM, Maggrah A, Reguly BJ, Harbottle A. Mitochondrial DNA deletions accurately detect endometriosis in symptomatic females of child-bearing age. Biomark. Med. 13(4), 291-306 (2019).

8. Pustylnikov S, Costabile F, Beghi S, Facciabene A. Targeting mitochondria in cancer: current concepts and immunotherapy approaches. Transl. Res. 202, 35-51 (2018).

9. Gammage PA, Frezza C. Mitochondrial DNA: the overlooked oncogenome? BMC Biol. 17(1), 53 (2019).

10. Maki JM, Robinson KA, Reguly BJ et al. Mitochondrial genome deletion aids in the identification of false- and true-negative prostate needle core biopsy specimens. Am. J. Clin. Pathol. 129(1), 57-66 (2008).

11. Harbottle A, Maki JM, Reguly BJ et al. Real-time polymerase chain reaction analysis of a 3895-bp mitochondrial DNA deletion in epithelial swabs and its use as a quantitative marker for sunlight exposure in human skin. BJD 63(6), 1291-1295 (2010).

12. Zhu W, Qin W, Sauter ER. Large-scale mitochondrial DNA deletion mutations and nuclear genome instability in human breast cancer. Cancer Detect. Prev. 28(2), 119-126 (2004).

13. Fliss MS, Usadel H, Caballero OL et al. Facile detection of mitochondrial DNA mutations in tumors and bodily fluids. Science 287(5460), 2017-2019 (2000). 
14. García-Lepe UO, Bermúdez-Cruz RM. Mitochondrial genome maintenance: damage and repair pathways. In: DNA Repair-An Update. Mognato M Ed. Intechopen, London, UK. (2019).

15. Nie H, Shu H, Vartak R et al. Mitochondrial common deletion, a potential biomarker for cancer occurrence, is selected against in cancer background: a meta-analysis of 38 studies. PLoS ONE 8(7), e67953 (2013).

16. Shizhen Z, Pin Q, Fuxiang L et al. Association of mitochondrial DNA variations with lung cancer risk in a Han Chinese population from southwestern China. PLoS ONE 7(2), e31322 (2012). 